\title{
COMPORTAMENTO ALIMENTAR DE AVES EM ALCHORNEA GLANDULOSA (EUPHORBIACEAE) EM RIO CLARO, SÃO PAULO
}

\author{
Renata de Melo Valente ${ }^{1}$
}

\begin{abstract}
FORAGING BEHAVIOR OF BIRDS AT ALCHORNEA GLANDULOSA (EUPHORBIACEAE) IN RIO CLARO, SÃO PAULO. Fourteen bird species were observed visiting one specimen of Alchornea glandulosa Poepp., 1845, at the Horto Florestal Navarro de Andrade, Rio Claro, São Paulo State, Brazil. Among them, three species seem to be the most efficient seed dispersers of A. glandulosa at the study site: Myiodinastes maculatus (Müller, 1776), Empidonomus varius (Vieillot, 1818) e Tyrannus melancholicus Vieillot, 1819. Besides to swallow fruit whole, these birds contributed with $68.5 \%$ of the total feeding visits. A. glandulosa fruit are not the mainly part of the diet of any one of the observed bird species, since they do not fit in the frugivorous specialized category. However, as almost all the observed individuals swallow fruit whole, it is suppose that they promote an efficient dispersion of their seeds.
\end{abstract}

KEYWORDS. Frugivory, Alchornea glandulosa, birds, behavior, Brazil.

\section{INTRODUÇÃO}

Tanto em regiões tropicais como em regiões temperadas, é freqüente a dispersão de sementes por vertebrados (STEBBins, 1971; JANZEN, 1983). Ornitólogos têm estudado a relação entre aves que se alimentam de frutos e as árvores que fornecem esses frutos como fonte de alimento (SNOw, 1971; McKey, 1975; Howe \& Estabrook, 1977; Fleming, 1979). Nesse contexto, muito tem sido discutido a respeito da diferença entre aves frugívoras especializadas, ou seja, aquelas que se alimentam de frutos de alta qualidade, ricos em gorduras e proteínas, e que dispersam as sementes de forma mais eficaz, e aves frugívoras oportunistas, que se alimentam de frutos menos nutritivos, que oferecem principalmente carboidratos (SNOw, 1981; FuENTES, 1994). Os frutos consumidos pelas aves especializadas, segundo SNow (1981), normalmente são maiores e possuem uma ou duas sementes grandes, podendo ser drupas ou arilados. Os frutos consumidos por aves oportunistas geralmente são menores e contêm muitas sementes pequenas. Outros fatores, como as diferentes estratégias de produção de frutos, incluindo a fenologia, também estão associados com a determinação desses dois tipos de frugivoria e, conseqüentemente, com a dispersão de sementes (SNOw, 1976).

Alchornea glandulosa Poepp., 1845, é uma planta pertencente à família Euphorbiaceae, que possui cerca de 5000 espécies distribuídas por todo o mundo (WILLIS, 1973). É uma espécie dióica, com cerca de 10 a $20 \mathrm{~m}$ de altura, e que no Brasil distribuise do Rio de Janeiro e Minas Gerais até o Rio Grande do Sul. É perenifólia, heliófila, pioneira, freqüente em beiras de rios e em planícies aluviais da Floresta Pluvial Atlântica, e cujos frutos amadurecem em meados de setembro a outubro e de dezembro a janeiro (LoRENZI, 1992). SNOw (1971) sugeriu que os frutos em geral são colhidos pelas aves de poucas maneiras diferentes. Segundo HERRERA \& JORDANO (1981), eles podem ser colhidos em vôo ou estando a ave empoleirada. Apesar de sua ampla distribuição e, por se tratar

1. Departamento de Zoologia, Museu Paraense Emílio Goeldi, Caixa Postal 399, 66040-170, Belém, PA, Brasil. 
de uma planta pioneira, de sua importância na recomposição de áreas degradadas, pouco se sabe sobre os mecanismos de frugivoria e dispersão de $A$. glandulosa. SNow (1981) considerou que os frutos do gênero Alchornea são comidos tanto por aves frugívoras especializadas quanto oportunistas e comentou que nenhum gênero da família Euphorbiaceae teria muita importância no sentido de contribuir com a maior parte da dieta de qualquer ave frugívora especializada.

Objetiva-se identificar as aves que visitam A. glandulosa no Horto Florestal Navarro de Andrade, em Rio Claro, registrar o comportamento alimentar daquelas espécies que ingeriram frutos e avaliar a importância das aves na dispersão das sementes de $A$. glandulosa.

\section{MATERIAL E MÉTODOS}

O Horto Florestal Navarro de Andrade (22 $25^{\prime}$ 'S e 47 $33^{\prime} \mathrm{W}$ ) situa-se a leste da área urbana do município de Rio Claro, São Paulo, Brasil. O clima da região, segundo KöPPEN (1948), é mesotérmico, de inverno seco, com duas estações definidas, uma chuvosa, de outubro a março, e outra seca, de abril a setembro. No período chuvoso, a precipitação média é de $1200 \mathrm{~mm}$ e no período seco é de aproximadamente $200 \mathrm{~mm}$. A área total do Horto é de 2314 ha e encontra-se dividida em talhões (PlattinEti-Júnior, 1978). O talhão em que foi feito o estudo é o de número 54, caracterizado por ser um sub-bosque de Eucalyptus tereticornis Sm., 1793, com área de 19,70 ha. Além dos eucaliptos, foram introduzidas algumas espécies, como Lecythis pisonis Cambess., 1829 (sapucaia) e Aspidosperma polyneuron Mull. Arg., 1860 (peroba), tendo ocorrido ainda regeneração natural de outras, como Nectandra megapotamica (Spreng.) Mez, 1903 (canelinha). Nesta área, foi observado um indivíduo de $A$. glandulosa em frutificação, com cerca de $12 \mathrm{~m}$ de altura, localizado à beira do Ribeirão Claro, o qual atravessa o Horto no sentido norte-sul (PlATTINETI-JúNIOR, 1978). As aves foram observadas e identificadas com o auxílio de binóculo, utilizando-se a classificação taxonômica adotada por SıcK (1997). As observações foram feitas nos dias $8,12,13,15,16$ e 18 de outubro de 1996 , pela manhã, totalizando 20 horas de observação. Foram registradas as espécies de aves que visitaram a planta, o número de visitas de alimentação e o comportamento alimentar daquelas que consumiram os frutos. As terminologias utilizadas para designar as técnicas de obtenção dos frutos seguem REMSEN \& RoBSON (1990). A freqüência das visitas foi calculada dividindo-se o número total de visitas de cada espécie, pelo total de "horas-planta" de observação, ou seja, a observação da planta durante 60 minutos (SILVA, 1988).

Frutos maduros foram coletados para análise morfológica e medida de suas partes.

\section{RESULTADOS}

Os frutos de $A$. glandulosa são cápsulas, normalmente biloculadas, do tipo drupa esquizocárpicas e deiscentes. Apresentaram comprimento médio de $8,7 \mathrm{~mm}$ e largura média de 5,9 mm $(\mathrm{n}=12)$. As sementes mediram 4,45 x 4,45 mm $(\mathrm{n}=10)$. Os frutos abrem-se quando maduros, expondo as sementes que são cobertas por um arilo vermelhovivo. Quando os frutos estão amadurecendo, a planta passa por um processo de perda total de suas folhas. Dessa forma, as infrutescências, que tendem a localizar-se nas pontas dos galhos, ficam mais expostas.

Quatorze espécies de aves, incluídas em 8 famílias ou subfamílias, visitaram o indivíduo de $A$. glandulosa durante o período de observação (tab. I). Quanto aos métodos de obtenção dos frutos pelas aves, foram observados dois tipos básicos de técnicas de captura: em vôo ou estando a ave empoleirada. Algumas espécies empregaram os dois tipos, embora um deles sempre fosse predominante em relação ao outro (tab. I).

Myiodinastes maculatus, Tyrannus melancholicus e Empidonomus varius utilizaram predominantemente a técnica "adejar", isto é, parar em frente ao fruto, enquanto batem as asas ("sally-hover"). Quando manifestaram o método de pegar o 
Tabela I. Espécies de aves registradas visitando um indivíduo de Alchornea glandulosa Poepp., 1845, no Horto Florestal Navarro de Andrade, Rio Claro, em 20 horas-planta de observação. Freqüência, número de visitas/horaplanta. Estratégia de obtenção dos frutos: p, a partir de poleiro; v, em vôo (em ordem decrescente de frequiência de observação). Dieta: f, frugívora; g, granívora; i, insetívora (inclui outros invertebrados); 1, folhívora; n, nectarívora (em ordem de importância). *Espécies que não se alimentaram dos frutos.

\begin{tabular}{|c|c|c|c|c|c|}
\hline $\begin{array}{l}\text { Famílias ou } \\
\text { Subfamílias }\end{array}$ & Espécies & $\begin{array}{l}\text { Número } \\
\text { de visitas }\end{array}$ & Freqüência & Estratégia & Dieta \\
\hline Columbidae & Leptotila rufaxilla (Richard \& Bernard, 1712) & 1 & 0,05 & $\mathrm{p}$ & $\mathrm{gf}$ \\
\hline \multirow[t]{2}{*}{ Cuculidae } & * Piaya cayana (Linnaeus, 1766) & 1 & 0,05 & & if \\
\hline & * Piaya minuta (Vieillot, 1817) & 1 & 0,05 & & if \\
\hline \multirow[t]{6}{*}{ Tyrannidae } & Myiozetetes cayanensis (Linnaeus, 1766) & 1 & 0,05 & V & ifg \\
\hline & Myiodinastes maculatus (Müller, 1776) & 39 & 1,95 & $\mathrm{~V} / \mathrm{p}$ & if \\
\hline & Empidonomus varius (Vieillot, 1818) & 15 & 0,75 & $\mathrm{~V} / \mathrm{p}$ & if \\
\hline & * Tyrannus savana (Linnaeus, 1766) & 1 & 0,05 & & if \\
\hline & T. melancholicus Vieillot 1819 & 13 & 0,65 & $\mathrm{~V} / \mathrm{p}$ & if \\
\hline & Pachyramphus validus (Vieillot, 1816) & 1 & 0,05 & $\mathrm{p}$ & if \\
\hline Turdinae & * Turdus leucomelas Vieillot 1818 & 1 & 0,05 & & $\mathrm{fi}$ \\
\hline Vireonidae & Vireo olivaceus (Vieillot, 1808) & 18 & 0,90 & $\mathrm{p}$ & fi \\
\hline \multirow[t]{3}{*}{ Thraupinae } & Thraupis sayaca (Linnaeus, 1766) & 3 & 0,15 & $\mathrm{p}$ & finl 1 \\
\hline & Tangara cayana (Linnaeus, 1766) & 2 & 0,10 & $\mathrm{p}$ & $\mathrm{fi}$ \\
\hline & Dacnis cayana (Linnaeus, 1766) & 12 & 0,60 & $\mathrm{p}$ & if $n$ \\
\hline
\end{tabular}

fruto a partir de um poleiro (cinco vezes para M. maculatus, uma vez para T. melancholicus e duas para E. varius), as duas primeiras espécies o fizeram utilizando como tal um galho que se encontrasse à frente do fruto. Assim, empregaram a técnica "alcançar", isto é, alongar o pescoço em direção ao fruto, retirando-o com o bico ("reach-out"). E. varius, entretanto, utilizou o próprio galho do fruto como poleiro, retirando-o sem estender o corpo ou assumir uma posição especial, empregando a técnica "pegar" ("glean"). As espécies que apanharam frutos apenas a partir de um poleiro, sempre o fizeram utilizando o próprio galho da infrutescência da qual estavam se alimentando.

Todas as aves pareceram engolir os frutos de $A$. glandulosa inteiros, ou seja, a semente com o arilo. Entretanto, Thraupis sayaca manifestou o comportamento de mandibular brevemente os frutos antes de ingeri-los e $V$. olivaceus, numa ocasião, estando no mesmo galho da infrutescência da qual se alimentava, pareceu dar bicadas, tendo deixado cair o fruto em seguida. Em todas as outras visitas de alimentação, V. olivaceus arrancou e engoliu o fruto inteiro, posicionando-se, algumas vezes, de cabeça para baixo, empregando a técnica "pendurar para baixo" ("hang-down"). Este posicionamento também foi adotado por T. melancholicus quando se alimentou a partir de poleiro, e foi observado uma vez para T. sayaca.

As espécies que visitaram a planta, mas não se alimentaram (tab. I), provavelmente estavam à caça de insetos. T. melancholicus, que freqüentemente foi vista se alimentando dos frutos, em certa ocasião visitou a árvore mas apenas tentou capturar insetos, não tendo consumido nenhum fruto.

\section{DISCUSSÃO}

Os frutos de A. glandulosa apresentam características morfológicas que se encaixam na "síndrome" de dispersão por aves, ou ornitocoria, segundo a descrição de PIJL (1972). 
Isto se dá principalmente pelo fato da semente ser envolta por um arilo vermelho, o qual é exposto quando o fruto amadurece e abre-se.

A eficiência de um dispersor pode ser avaliada através de fatores comportamentais, tais como freqüência de visitas e forma como o fruto é trabalhado antes da ingestão, entre outros (Schupp, 1993). Nesse contexto, M. maculatus, E. varius e V. olivaceus são provavelmente as espécies mais importantes no processo de dispersão das sementes de A. glandulosa, pois contribuem com $68,5 \%$ do total de visitas, além de engolirem os frutos inteiros. As duas primeiras espécies, juntamente com T. melancholicus, pertencentes à família Tyrannidae, empregaram principalmente a técnica de apanhar o fruto adejando, o que pode representar, segundo MARCONDES-MACHADO et al. (1994), um comportamento inato na captura de diversos itens alimentares.

Os frutos que servem de alimento para as aves podem ser engolidos inteiros, comidos aos pedaços ou mandibulados (Moermond \& Denslow, 1985). Dependendo de quanto são mandibulados, as sementes podem ou não ser esmagadas (MoERMOND, 1983).O comportamento de mandibular, exibido por T. sayaca, também tem sido observado quando a mesma se alimenta dos frutos de outras espécies, como Ficus microcarpa L. f., 1782 (Moraceae) (Marcondes-Machado et al., 1994), Ficus clusiifolia Schott (Moraceae) (ARgel-DE-Oliveira \& Figueiredo, 1996) e Trema micrantha (L.) Blume, 1856 (Ulmaceae) (Argel-DE-Oliveira et al., 1996). Como foi sugerido por esses autores, este tipo de processamento provavelmente não chega a danificar as sementes, e T. sayaca também pode ser considerada uma dispersora eficaz das sementes de A. glandulosa. Apenas Leptotila rufaxilla, que é uma ave granívora, representa uma provável exceção a esse modelo. Como utiliza-se deste recurso a fim de obter as sementes como principal fonte de alimento, é considerada destruidora das mesmas, já que são destruídas em seu tubo digestivo (MoERMond \& Denslow, 1985).

Das espécies que foram vistas alimentando-se dos frutos de $A$. glandulosa no Horto de Rio Claro, nenhuma se enquadra na categoria de frugívoros especializados, segundo Snow (1981). As espécies observadas encaixam-se na categoria de aves oportunistas, já que os frutos não constituem um componente predominante em suas dietas (SICK, 1997). Entretanto, como engolem o fruto inteiro e não permanecem muito tempo na árvore, podem ser consideradas dispersoras efetivas das sementes desta espécie.

Segundo SNow (1981), uma análise química dos frutos das Euphorbiaceae, provavelmente revelaria que eles possuem um baixo valor nutritivo para as aves. Este fator talvez explique o fato de que os frutos das espécies dessa família não têm sido encontrados como parte principal da dieta de nenhuma ave frugívora especializada. Moermond \& Denslow (1985) chamam a atenção para o fato de que muitos frutos que em determinados períodos são abundantes, visíveis e de fácil acesso, podem ser explorados ocasionalmente por muitas aves, já que constituem uma fonte de energia alternativa e farta, muito embora sem grande valor nutritivo. Através dessa estratégia, típica de plantas de matas secundárias (HARPER et al., 1970; PIJL, 1972), as sementes podem ser dispersas eficazmente por aves principalmente insetívoras ou por aves frugívoras não especializadas. De fato, A. glandulosa apresentou um pico de frutificação no qual recebeu a visita de muitas espécies e muitos indivíduos ao mesmo tempo, inclusive nas horas mais quentes do horário de observação, isto é, no final da manhã. Porém, a maior parte das observações ocorreu num período em que houve um decréscimo no ritmo de 
amadurecimento dos frutos, o que provocou uma diminuição considerável no número de espécies visitantes.

Segundo McKey (1975), frugívoros oportunistas são típicos de áreas mais abertas ou perturbadas, e não possuem um padrão regular de visitas. Várias espécies podem ter se alimentado dos frutos de $A$. glandulosa apenas quando esta representou um recurso superabundante e que exigiu esforço mínimo das aves. Segundo WiLlis (1966), o nível de competição interespecífica pode ser reduzido em plantas que apresentem essas características. Um estudo que seja realizado no período de pico de frutificação dessa espécie, entre final de setembro e início de outubro, provavelmente revelará um número bem maior de espécies de aves que utilizam-se de seus frutos como recurso alimentar.

Agradecimentos. A Ivan Sazima (UNICAMP) pelas sugestões, a Ivonne Gajardo pela ajuda prestada durante o trabalho de campo, a Márcia Santos pela correção do abstract e a dois revisores anônimos pela leitura crítica do manuscrito.

\section{REFERÊNCIAS BIBLIOGRÁFICAS}

Argel-de-Oliveira, M. M.; Castiglioni, G. D. A. \& Souza, S. B. 1996. Comportamento alimentar de aves frugívoras em Trema micrantha (Ulmaceae) em duas áreas alteradas do sudeste brasileiro. Ararajuba, Londrina, 4:51-55.

Argel-De-Oliveira, M. M. \& Figueiredo, R. A. 1996. Aves que visitam uma figueira isolada em ambiente aberto, Espírito Santo, Brasil. Iheringia, Sér. Zool., Porto Alegre, (80):127-134.

Fleming, T. H. 1979. Do tropical frugivores compete for food? Am. Zool., Kansas, 19:1157-1172.

FUENTES, M. 1994. Diets of fruit-eating birds: what are the causes of interspecific differences? Oecologia, Berlin, 97:134-142.

Harper, J. L.; Loveli, P. H. \& Moore, K. G. 1970. The shapes and sizes of seeds. Ann. Rev. Ecol. Syst., Palo Alto, 1:327-356.

Herrera, C. M. \& Jordano, P. 1981. Prunus mahaleb and birds: the high-efficiency seed dispersal system of a temperate fruiting tree. Ecol. Monogr., New York, 51:203-218.

Howe, H. F. \& Estabrook, G. F. 1977. On interespecific competition for avian dispersers in tropical trees. Am. Nat., Chicago, 111:817-832.

Janzen, D. H. 1983. Dispersal of seeds by vertebrate guts. In: Futuyama, D. J. \& Slatkin, M. eds. Coevolution. Sunderland, Sinauer.p. 232-262.

KöPPEN, W. 1948. Climatologia. Buenos Aires, Fondo de Cultura Econômica. 478p.

LORENZI, H. 1992. Árvores Brasileiras: manual de identificação e cultivo de plantas e árvores nativas do Brasil. Nova Odessa, Plantarum. 352 p.

Marcondes-Machado, L. O.; Paranhos, S. J. \& Barros, Y. M. 1994. Estratégias alimentares de aves na utilização de frutos de Ficus microcarpa (Moraceae) em uma área antrópica. Iheringia, Sér. Zool., Porto Alegre, (77):57-62.

McKey, D. 1975. The ecology of coevolved seed dispersal systems. In: Gilbert, L. E. \& Raven, P. H. eds. Coevolution of animals and plants. Austin, University of Texas. p. 159-191.

Moermond, T. C. 1983. Suction-drinking in tanagers and its relation to fruit handling. Ibis, London, 125:545-549.

Moermond, T. C. \& Denslow, J. L. 1985. Neotropical avian frugivores: patterns of behavior, morphology, and nutrition, with consequences for fruit selection. Ornithol. Monogr., Washington, 36:865-897.

Plattineti-Júnior, A. 1978. O Horto Florestal Navarro-de-Andrade. In: Machado, I. L. coord. Rio Claro Sesquicentenária. Rio Claro, Museu Histórico e Pedagógico Amador Bueno da Veiga. p. 295-308.

PIJL, L. VAR DER. 1972. Principles of dispersal in higher plants. 2. ed. New York, Springer-Verlag. 215 p.

Remsen, J. V., JR. \& Robson, S. K. 1990. A classification scheme for foraging behavior of birds in terrestrial habitats. Stud. Avian Biol., Los Angeles, 13:144-160.

Schupp, E. W. 1993. Quantity, quality and the effectiveness of seed dispersal by animals. Vegetatio, Haia, 107/ 108:15-29.

Sick, H. 1997. Ornitologia Brasileira. Rio de Janeiro, Nova Fronteira. 862 p.

Silva, W. R. 1988. Ornitocoria em Cereus peruvianus (Cactacea) na Serra do Japi, Estado de São Paulo. Revta bras. Biol., Rio de Janeiro, 48:381-389. 
SNow, D. W. 1971. Evolutionary aspects of fruit-eating by birds. Ibis, London, 113:194-202.

_. 1976. The Webs of Adaptation. New York, Quadrangle. $176 \mathrm{p}$.

. 1981. Tropical frugivorous birds and their food plants: a world survey. Biotropica, Washington, 13:1-14.

STEBBins, G. L. 1971. Adaptive radiation of reproductive characteristics in angiosperms, II: seeds and seedlings. Ann. Rev. Ecol. Syst., Palo Alto, 2:237-260.

WiLLIS, E.O. 1966. Competitive exclusion and birds at fruiting trees in Western Colombia. Auk, Washington, 83:479-480.

WiLLIS, J. C. 1973. A dictionary of flowering plants and ferns. Cambridge, Cambridge Univ. 1245 p.

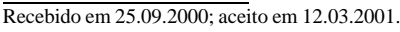

